\title{
Efficient Data Clustering Algorithms: Improvements over Kmeans
}

\author{
Mohamed Abubaker, Wesam Ashour \\ Dept. of Computer Engineering, Islamic University of Gaza, Gaza, Palestine \\ mabubaker@hotmail.com;washour@iugaza.edu.ps
}

\begin{abstract}
This paper presents a new approach to overcome one of the most known disadvantages of the well-known Kmeans clustering algorithm. The problems of classical Kmeans are such as the problem of random initialization of prototypes and the requirement of predefined number of clusters in the dataset. Randomly in itialized prototypes can often yield results to converge to local rather than global optimum. A better result of Kmeans may be obtained by running it many times to get satisfactory results. The proposed algorithms are based on a new novel definition of densities of data points which is based on the k-nearest neighbor method. By this definition we detect noise and outliers which affect Kmeans strongly, and obtained good initial prototypes from one run with automatic determination of $\mathrm{K}$ number of clusters. This algorithm is referred to as Efficient Initialization of Kmeans (EIKmeans). Still Kmeans algorithm used to cluster data with convex shapes, similar sizes, and densities. Thus we develop a new clustering algorith $m$ called Efficient Data Clustering Algorithm (EDCA) that uses our new definition of densities of data points. The results show that the proposed algorithms improve the data clustering by Kmeans. EDCA is able to detect clusters with different non-convex shapes, different sizes and densities.
\end{abstract}

Index Terms - Data Clustering, Random In itialization, Kmeans, K-Nearest Neighbor, Density, Noise, Outlier, Data Point

\section{Introduction}

Data clustering techniques are an important aspect used in many fields such as data mining [1], pattern recognition and pattern classification [2], data compression [3], machine learning [4], image analysis [5], and bioinformatics [6].

The purpose of clustering is to group data points into clusters in which the similar data points are grouped in the same cluster while dissimilar data points are in different clusters. The high quality of clustering is to obtain high intra-cluster similarity and low inter-cluster similarity.
The clustering problems can be categorized into two main types: fuzzy clustering and hard clustering. In fuzzy clustering, data points can belong to more than one cluster with probabilities [7] which indicate the strength of relationships between the data points and a particular cluster.

One of the most widely used fuzzy clustering algorithms is fuzzy c-mean algorithm [8]. In hard clustering, data points are divided into distinct clusters, where each data point can belong to one and only one cluster. The hard clustering is subdivided into hierarchical and partitional algorithms. Hierarchical algorithms create nested relationships of clusters which can be represented as a tree structure called dendrogram [9]. These algorith ms can be divided into agglomerative and divisive hierarchical algorith $\mathrm{ms}$. The agglomerative hierarchical clustering starts with each data point in a single cluster. Then it repeats merging the similar pairs of clusters until all of the data points are in one cluster, such as complete linkage clustering [10] and single linkage clustering [11]. The divisive hierarchical algorithm reverses the operations of agglomerative clustering, it starts with all data points in one cluster and repeats splitting large clusters into smaller ones until each data point belong to a single cluster such as DIANA clustering algorithm [12].

Partitional clustering algorithm divides the data set into a set of disjoint clusters such as Kmeans [13], PAM [12] and CLARA [12].

One of the most well-known unsupervised learning algorithms for clustering datasets is Kmeans algorithm [12]. The Kmeans clustering is the most widely used [14] due to its simplicity and efficiency in various fields. It is also considered as the top ten algorithms in data mining [15]. The Kmeans algorithm works as follows:

1. Select a set of initial $k$ prototypes or means throughout a data set, where $k$ is a user-defined parameter represents the number of clusters in the data set.

2. Assign each data point in a data set to its nearest prototypes $m$.

3. Update each prototype according to the average of data points assigned to it.

4. Repeat step 2 and 3 until convergence. 


$$
J=\frac{1}{n} \sum_{i=1}^{k} \sum_{x \in C_{i}}\left\|x-m_{i}\right\|^{2}
$$

The Kmeans updates their prototypes iteratively to minimize the following criterion function:

Where data set $D$ contains $n$ data points or objects $x_{1}, \ldots, x_{n}$ such as each data point is $d$ dimensional vector in $R^{d}$, and $m_{i}$ is the prototype of cluster $C_{i}$, and $k$ is the given number of clusters.

The main advantages of Kmeans algorithm are its simplicity to be implemented and its efficiency. However, it has several drawbacks:

- The number of clusters in a given data set should be known in advance.

- The result strongly depends on the initial prototypes.

- It is applicable when the mean of data is defined.

- Sensitivity to noise and outliers.

- Dead prototypes or Empty clusters.

- Converge to local optima.

- It is defined for globular shaped, similar size and density clusters.

A number of kernel methods have been proposed in recent years [16-19] to increase the separable of clusters. In the kernel Kmeans algorithm all data points are mapped, before clustering, to a higher dimensional feature space by using a kernel function. Then the Kmeans algorith $m$ is applied in the new feature space to identify clusters. Recently, [20,21] proposed a novel of new clustering algorithms that converge to a better solution (less prone to finding a local minimu m because of poor initialization) than both standard Kmeans and a mixture of experts trained using the EM algorithm.

In this paper, we modify the non-parametric density estimation based on $\mathrm{k}_{\mathrm{n}}$-nearest neighbors algorith $\mathrm{m}$ [22]. So, our proposed algorithm is robust to noise and outliers, automatically detects the number of clusters in the data set, and selects the most representative dense prototypes to be initial prototypes even if the clusters are in different shapes with different densities.

To compute the $\mathrm{k}_{\mathrm{n}}$-nearest neighbors algorithm of a data point $x_{i} \in D$, we center a ball cell about $x_{i}$ and let it grows until it captures the predefined number $k_{n}$ of data points. Let $R\left(N\left(x_{i}\right)\right)$ represents the radius of the ball which is the distance from $x_{i}$ to its farthest neighbor in its $N\left(x_{i}\right)$, where $N\left(x_{i}\right)$ represents a set of data points in the $k_{n}$ neighborhood of $x_{i}$. Then the density of $x_{i}$ is defined as [22,23]:

$$
\operatorname{den}\left(x_{i}\right)=\frac{k_{n}}{n \cdot V\left(R\left(N\left(x_{i}\right)\right)\right)}
$$

Where $V(r)$ represents the volume of ball of radius $r$ in $R^{d}$, and $V(r)=2 \pi^{d / 2} r^{d} / d \Gamma(d / 2)$.

The clustering algorithms that are based on estimating the densities of data points are known as density-based. One of the basic density based clustering algorithm is DBSCAN [24]. It defines the density by counting the number of data points in a region specified by a predefined radius known as Eps around the data point. If a data point has a number greater than or equal to predefined minimum points, then this point is treated as a core point. Non-core data points that do not have a core data point within the predefined radius are treated as noise. Then the clusters are formed around the core data points and are defined as a set of density-connected data points that is maximal with respect to density reachability. DBSCAN may behave poorly due it is weak definition of data points' densities and it is globally predefined parameters.

\section{Related Works}

There are several prototypes initialization methods have been introduced for the classical Kmeans algorithms. In [25] the prototypes are chosen randomly from the data set which considers the simplest and most common initialization method. The Min max [26] selects the first prototype randomly $m_{1}$ then the $i^{\text {th }}$ prototype $m_{i}$ is selected to be the data points with largest minimum distance to the previously selected prototypes. One of the drawbacks of this method is that it is sensitive to outliers thus it selects the outliers in the data set. Kmeans++ [27] in which the first prototype $m_{i}$ is selected randomly $m_{l}$ then the $\mathrm{i}^{\text {th }}$ prototype is selected to be $x^{\prime} \in D$ with probability of $d\left(x^{\prime}\right)^{2} / \sum_{x \in D} d(x)^{2}$, where $d(x)$ denotes the shortest distance from data point $x$ to the closet prototype already chosen. Al-Daoud in [28] proposed an algorithm to initialize the prototypes of Kmeans which finds a set of medians extracted from the dimension with maximum variance to be the initial prototypes. The use of median in this method makes the algorith m sensitive to outliers. Gan, $\mathrm{Ma}$, and $\mathrm{Wu}$ in [9] introduced a validity measure to determine the number of clusters in Kmeans algorithm. This method depends on calculating the intra-cluster distances $M_{\text {intra }}$ which is defined as in (1) and the inter-cluster distances $M_{\text {inter }}$ which is the minimum distance between pair of prototypes among all prototypes. Then the validity measure is defined as: $V=M_{\text {intra }} / M_{\text {inter }}$. Obviously a good result shall have a small intra-cluster distances and a large inter-cluster distances, thus $V$ is minimized. To determine $V$ we shall apply Kmeans algorith $\mathrm{m}$ fro $\mathrm{m} \mathrm{k}=2$ up to $K_{\max }$ and for each we calculate the validity measure and choose the $k$ that corresponds to the minimum value of $V$.

\section{Motivations}

The Kmeans algorithm considered as one of the top ten algorithms in data mining [15]. A lot of researches and studies have been proposed due to its simplicity and efficiency. These efforts have focused on finding 
possible solutions to one or more of the limitations that have been identified previously. One of the solutions to the initial prototypes sensitivity can be found in [29] where they defined new criterion functions for Kmeans and they proposed three new algorithms: weighted Kmeans, inverse weighted Kmeans [30] and inverse exponential Kmeans [31]. Other improvements of Kmeans focus on its efficiency where the complexity of Kmeans involves the data set size, number of dimensions, number of clusters and the number of iteration to be converged. There are many works to reduce the computational load and make it more fast such as in [32-34]. Asgharbeygi and Maleki in [23] proposed a new distance metric which is the geodesic distance to ensure resistance to outliers. Several works have been introduced to extend the use of means for numerical variables, thus Kmeans can deal with categorical variables such as in $[35,36]$.

Our proposed EI-Kmeans algorithm focuses on classical Kmeans itself. We want to in itialize prototypes from the first run on compete positions throughout the data set that yields good results. EI-Kmeans also solves one of the most difficult problems in the data clustering which is the determination of the number of clusters in the data set in advance. The determination of nu mber of clusters depends on our new defined density of data points that can eliminate noise and outliers from the data set. The proposed EDCA tries to benefit from the proposed EI-Kmeans to develop a new clustering algorithm that is able to detect clusters with different non-convex shapes, different sizes and densities in which Kmeans cannot give good results in these types of data sets.

\section{Proposed Algorithms}

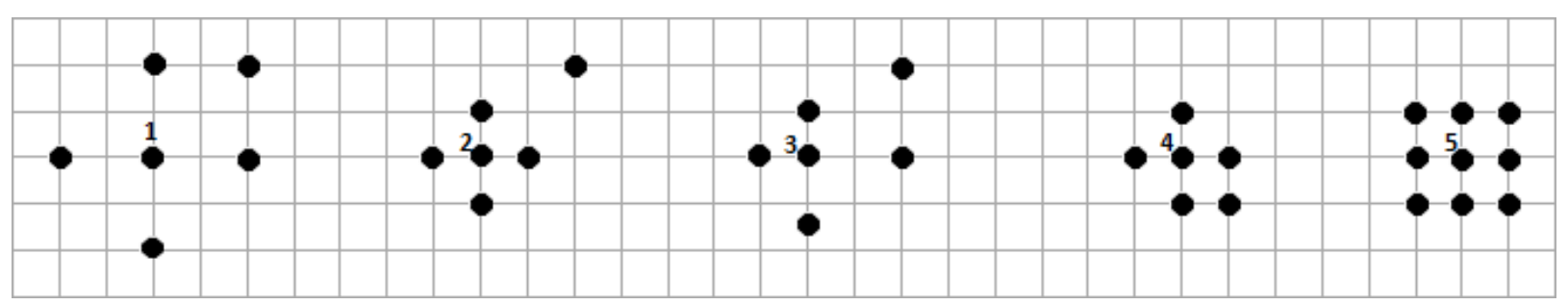

Fig. 1: Illustration example

We want to examine the density of the numbered data points from 1 to 5 with respect to $k_{n}=6$ including the examined data point. By applying (2), the density of data points 1,2 , and 3 is the same and equals to 0.007 . And the density of data points 4 and 5 equals to 0.029 . It is obvious that each numbered data point has a different density with data point number 5 is the densest one and data point number 1 has a least density. It is true that (2) does not take into account the actual number of data points within ball cell about examined data points with respect to $k_{n}$. If we try to engage the
The proposed algorithms define a new definition for the density of data points throughout the data set. This new definition mentions the drawback of (2) which is based on $\mathrm{k}_{\mathrm{n}}$-nearest neighbors density estimation $[22,23]$. EI-Kmeans calculates the density of each data point in the given data set, and then it sorts the data points in descending order according to their densities. The first densest point is selected as the first prototype. Then the list of sorted data points is investigated to get the next candidate prototypes. However, we shall take care that the selected prototype does not have a direct connectivity with the previously selected prototypes. We define two versions of EI-Kmeans algorithm. The first version takes $k_{n}$ the number of nearest neighbors, and the number of clusters $k$ in the data set as input parameters. Then the sorted list of data points is investigated until the number of obtained prototypes reached the specified $k$ then the algorithm is aborted. The second version takes $k_{n}$ the number of nearest neighbors as the only input parameter and obtains both the number of clusters and the prototypes in parallel. We also propose another version which is an improvement of our second version. However, this third version considers as a new clustering algorithm. This new algorith $\mathrm{m}$ is referred to as Efficient Data Clustering Algorithm (EDCA).

The following subsections describe the proposed algorithms in details:

\subsection{New definition of density}

We derived our new formula of density based on the drawbacks of (2).

To illustrate this drawback, let us consider the simple data set example in which it contains 33 data points distributed as shown in Figure 1. Assume grid is a unit distance. sum of Euclidean distances from the examined data point to all of its neighbors defined by $k_{n}$. Then we have the formula for density of data points: $\operatorname{den}(x)=N_{t} / \sum_{y \in N(x)} d(x, y)$ where $N_{t}$ represents the actual number of data points in the neighborhood of data point $x$ with respect to $k_{n}, N(x)$ is a set of data points in the neighborhood of data point $x$ with respect to $k_{n}$, and $d(x, y)$ is the Euclidean distance from data point $x$ to data point $y$. Then the density of point numbered 1 is 0.55 , density of data point nu mbered 2 is 
0.88 , density of data point numbered 3 is 0.72 , density of data point numbered 4 is 1.11 , and density of data point numbered 5 is 0.93 . Again this formula fails to specify the density of data points accurately. Then we put our new formula of density based on the density estimation of (2). The density of data point $x$ is defined as:

$$
\operatorname{density}(x)=\frac{N_{t}}{n \cdot V \sum_{y \in N_{n}(x)} d(x, y)}
$$

Where $N_{t}$ represents the actual number of data points in the neighborhood of data point $x$ with respect to $k_{n}, n$ is the total number of data points in the data set, $V$ is the volume defined as in (2), and $N_{n}(x)$ is a set of closest $k_{n}$ neighbors to point $x$.

According to (3), the density of point 1 is 0.66 , the density of data point 2 is 1.06 , the density of data point 3 is 0.87 , the density of data point 4 is 5.34 , and the density of data point 5 is 8.02 .

\subsection{EI-Kmeans algorithm}

Let $D$ be a data set of $n$ d-dimensional data points $x_{1}, \ldots, x_{n}$. Then we want to find the best candidate data points to be the initialized prototypes. Thus we can perform the Kmeans clustering algorithm on the given data set for obtaining a good result of clustering data points. The proposed algorithm solves the problem of considering the number of clusters of the data set in advance. And it solves the problem of bad initialized prototypes that may yield the algorith $m$ to converge to local optima or to get empty clusters [29]. EI-Kmeans algorithm uses our new formula of density defined in (3). EI-Kmeans searches the data set for the densest points which are strong candidate prototypes. We take care that the given data set may contain different clusters that have different densities, thus a number of densest points can present in the same cluster.

Let the set $P$ consists of initial prototypes of data set and it is initialized to be empty. We compute the density for each data point in the data set. Then we sort the data point according to their densities in descending order. The first data point in this sorted list is the densest data point in the entire data set. We choose this point to be the first in itialized prototypes. Then the set $P$ consists of this prototype. Now we want to examine the next densest data point in the sorted list in order. To avoid selecting prototypes that resides in the same cluster. We test the connectivity between the examined data point and each prototype in the set $P$. Thus, if there is no path between examined data point and each prototype in the set $P$, this examined data point is inserted in the set $P$. Then the next one which is not examined data point in the sorted list is tested to be an available prototype or not. This procedure is repeated until we obtain the desired prototypes. The path between pairs of data points is calculated, if exists, as a proactive scheme this means that we build a proximity matrix of 0 and 1 where 0 means that there is no direct connectivity between two data points and 1 means there is a direct connectivity between two data points. This is calculated according to a threshold value $\varepsilon$ which is calculated dynamically for the given data set. The value $\varepsilon$ defines the radius of the region in which direct connectivity is considered. We compute $\varepsilon$ for the given data set $D$ as follows:

$$
\varepsilon=\left[\frac{\operatorname{prod}(\max (x)-\min (x)) \cdot k_{n} \cdot \Gamma(0.5 d+1)}{n \cdot \sqrt{\pi^{d}}}\right]^{1 / d}
$$

Where $\max (x)$ treats the columns of $x$ as vectors, and returns a row vector containing the maximum element from each colu mn. The same thing for $\min$ but it returns the minimum element for each column. And $\operatorname{prod}(A)$ returns the product of elements of vector $A$.

Then we use the connectivity proximity matrix to find a path between pairs of data points. If the value of the path is infinity then there is no path between the given pairs of data points. The following two subsections describe two versions of EI-Kmeans algorithm.

\subsubsection{EI-Kmeans version 1}

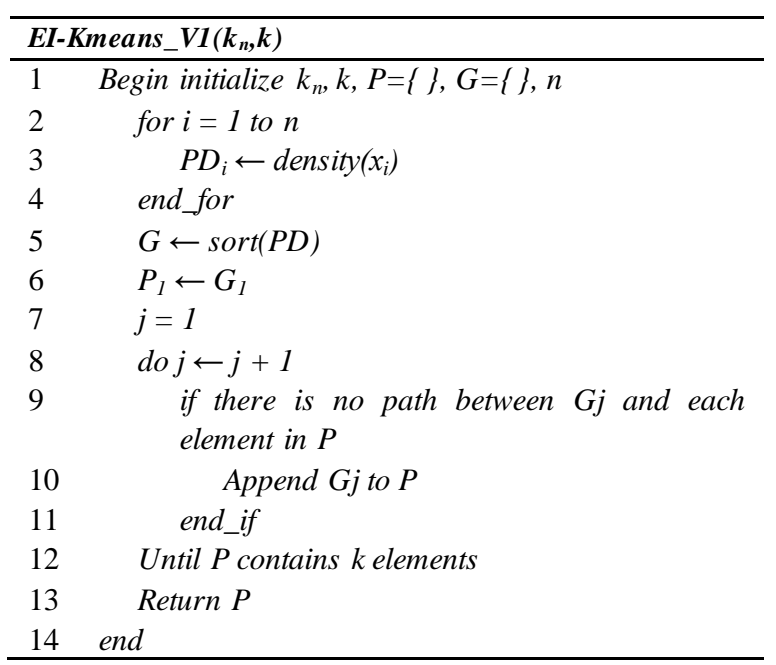

Fig. 2: EI-Kmeans algorithm version 1

The proposed version 1 of the algorithm takes two input parameters which are the $k_{n}$ number of nearest neighbors and $k$ the number of clusters in the data set. The output is the set of $k$ prototypes. Figure 2 shows the algorithm. The first step initializes two empty sets $P$ and $G$. The set $P$ contains indexes of chosen prototypes and the set $G$ is a sorted list of data points' indexes according to the density in descending order. And $n$ is the number of data points in the $D$ data set. For each data point in the data set, we compute the density of this data point according to (3) and store the results in an array list of Point Density (PD). Line 5 of the algorithm sorts obtained densities in descending order and put the result in the set $G$. Line 6 means that the first element in the set $G$ is selected as a first prototype. The first 
element of $G$ is the index of the densest data point in the data set. Then a counter $j$ points to the second densest data point and it is incremented by one for each loop throughout lines 8 to 12 . In each loop we test the pointed data point in the set $G$ if there is no path between this data point and each prototype in set $P$, we append the index of this data point to the set $P$. Otherwise, we increment the counter $j$ to test the next data point in $G$. These steps are repeated until $k$ prototypes are found. Then the algorithm breaks and returns the $k$ prototypes which they are considered the initial prototypes for the Kmeans clustering algorithm. Thus after obtaining these $k$ prototypes, we run the standard Kmeans clustering algorithm.

\subsubsection{EI-Kmeans version 2}

The second version of EI-Kmeans takes only one input parameter which is the $k_{n}$ number of nearest neighbors. Figure 3 presents the EI-Kmeans algorith m. If we mention our algorithm, then we mean the second version. Otherwise, it is stated explicitly.

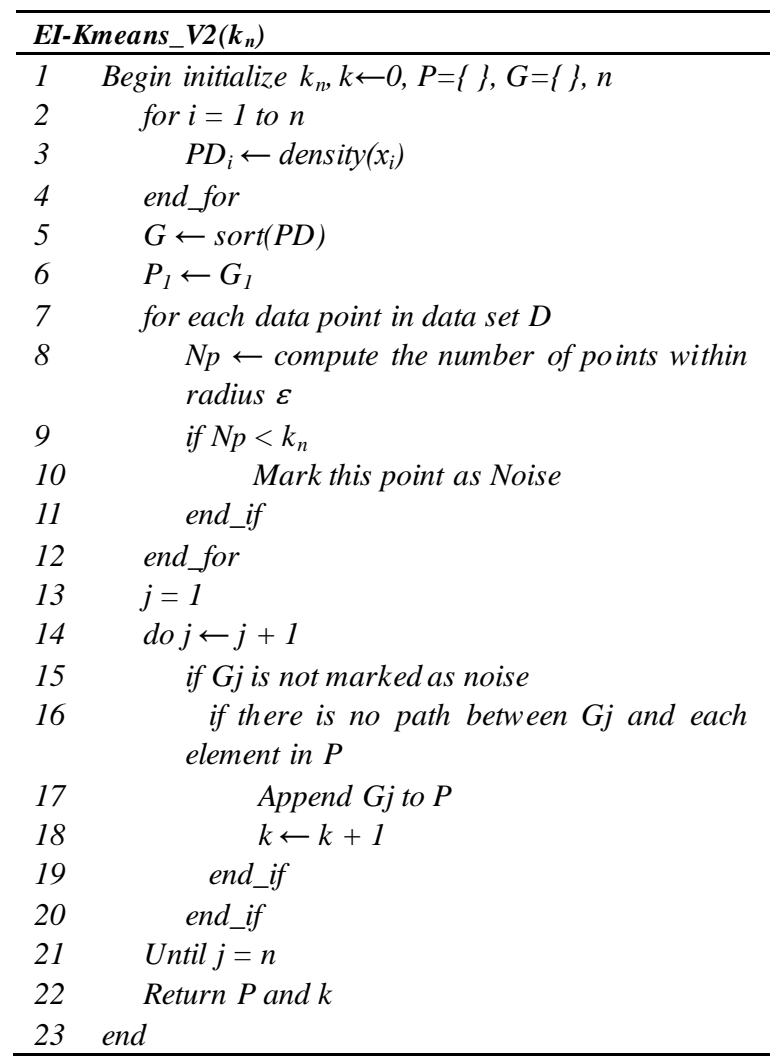

Fig. 3: EI-Kmeans algorithm version 2

We add a definition for noise points. These points are excluded from the computations and they often reside in the bottom of the set $G$. We investigate all of the data points in the set $G$ except the points marked as noise. The algorithm finds all of the possible prototypes in the entire data set. Thus the number of found prototypes indicates the number of clusters in the data set. The resulted prototypes are used as the initial prototypes for clustering the data set using Kmeans algorithm.

\subsection{A new clustering algorithm}

However, EI-Kmeans algorithm finds the best initialized prototypes locations entire the data sets, it inherent one of the limitations of Kmeans clustering algorith $\mathrm{m}$. This limitation is about the type of given data sets. In which the Kmeans algorithm has a problem of discovering clusters of different non-convex shapes, different sizes and densities. Thus we develop a new clustering algorithm to cope this limitation. This new algorithm is referred to as Efficient Data Clustering Algorithm (EDCA). EDCA is able to find clusters with different non-convex shapes, different sizes and densities. It also has a definition of noise and outliers. We benefit from our new definition of data points' densities to propose the EDCA.

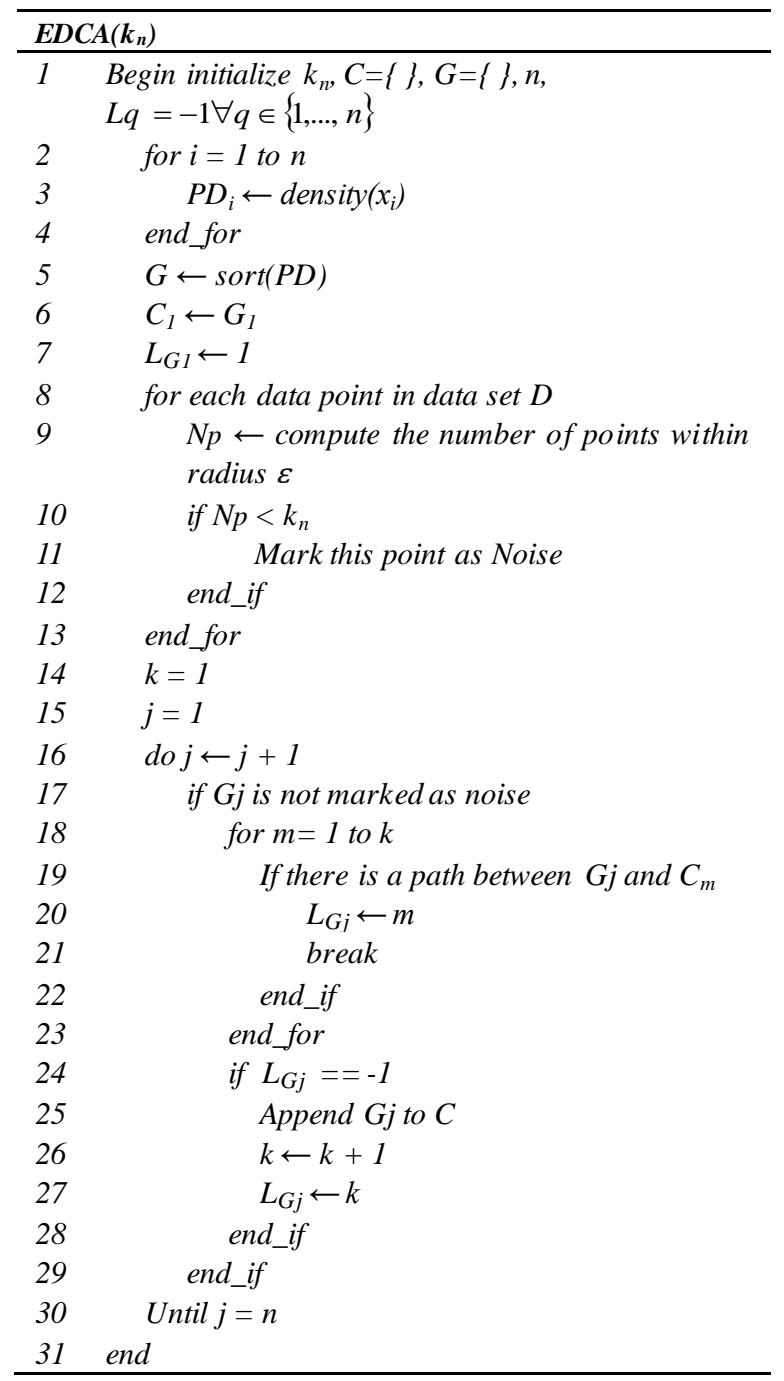

Fig. 4: EDCA algorithm.

Figure 4 shows the procedures of the algorithm. EDCA takes the number of nearest neighbors as the only input parameter. The first steps are the same as in our proposed EI-Kmeans algorith $\mathrm{m}$. We define the set $C$ which contains each cluster identification entire the given data set $D$. The most dense data point in a cluster is considered as the cluster identification. Thus the number of entries in the set $C$ represents the number of 
clusters in the data set. Initially, $C$ is empty. We denote the cluster label of the data point by $L q$. Initially all data points are assigned the label of -1 to indicate unassigned data point, that is $L q=-1 \forall q \in\{1, \ldots, n\}$. EDCA appends the index of the densest data point according to (3) to the set $C$ to be the first cluster identification and we label it to be in the first cluster. We use this point as the first reference to expand the cluster. Each data point in the data set is examined in order as in the set $G$ which is a set of data points' indexes arranged in descending order according to their densities. If the examined unlabeled data point has path reachability as described previously to one of the cluster identifications in the set $C$, we assign the label of that cluster identification to this examined data point. But if we have an unlabeled data point with no path to reach any of the cluster identifications in set $C$, then we know that the data point should belong to a new cluster. Thus we increment the number of recently obtained clusters and assign this cluster label to this data point.

Lines 2 to 4 are used to compute the density of each data point in the given data set $D$ according to our new definition of density. Line 7 as signs cluster label of 1 to the densest data point. Lines 8-13 figure out the noise and outliers in the data set. Thus our proposed algorith $\mathrm{m}$ is robust to noise and outliers. Line 14 defines $k$ to be the number of clusters in the data set and it is initially equal to 1 . Then we examine all the data points in the data set according to the sorted set $G$. Lines 18 through 23 assign a cluster label to the current data point. To do this, a loop is used to pass through all the elements in the set $C$. In case if there is a match, we assign a matched cluster label to the examined data point and exit the loop. If the algorithm fails to assign a label to the examined data point, the lines 24 through 28 appends this data point to the set $C$ to be new cluster identification then the value $k$ is incremented by one to reflect the so far number of obtained clusters. Then this update value of $k$ is assigned as a cluster label to this examined data point. These procedures are repeated until all the data points in the given data set are labeled.

\section{Simulation and Results}

We evaluated our proposed algorithms on several artificial and real data sets.

Artificial data set: We generate artificial two dimensional data sets, since the results are easily visualized. Consider the data set in Fig. 5(a) that consists of 300 data points with 5 clusters. Fig. 5(b) shows the locations of the prototypes from the first run of our algorithm. It is truly identifies that this data set contains 5 clusters. Thus our algorith $m$ does not need to have an input parameter of the number of clusters.

Figure 5(c) shows the clustering result when applying Kmeans using the prototypes obtained by EI-Kmeans. It is obvious that the clustering result in Figure 5(c) is true.
This is due to the fact that the initial prototypes are considered carefully, where each of the five prototypes catches one cluster. Figure 5(d) shows the location of the initial prototypes after 5 randomly runs of Kmeans, and then we select the result that minimizes the criterion function in (1). Figure 5(e) shows the failed best clustering result after 5 times running of Kmeans based on the initial prototypes of Figure 5(d) two clusters are identified correctly. However, there are two clusters are merged into one and one cluster is split to two clusters. This shows the overcome of convergence to local optima rather than to global optima. It is shown empirically that the Kmeans can truly identify the 5 clusters of data set in Figure 5(a) after 11 times running. Thus our proposed EI-Kmeans is stable and finds competent locations of the initial prototypes from the first run with one iteration to be converged and if we repeat the execution of the proposed algorithm more than once. The locations of the in itial p rototypes remain the same as in the first run. Due to the robust defined of data points' densities.

Since we base our proposed algorithm on our new definition of density, it is strongly recommend for testing it in a situation where the data set contains clusters with different densities. In Figure 6(a) we generate a data set with 400 data points and it has three clusters with different densities and sizes. When we apply our algorithm, it finds out the true number of clusters which is three, thus our algorithm automatically generates the number of clusters in this data set. About the initial prototypes, the proposed algorithm identifies the three prototypes from the first run into satisfactory positions as shown in Figure 6(b) which yield a true clustering result of three clusters which is shown in Figure 6(c) However, when we apply the Kmeans algorithms and inject it with the number of clusters. The clustering result has only two clusters and the third one is an empty cluster as shown in Figure 6(e). Thus the effect of the bad initialized prototypes makes the clustering behaves very poorly.

It is very important to verify our proposed algorithm in presence of noise. Figure 7(a) shows a generated four clusters data set with noise and outliers. We start our EI-Kmeans algorithm to discover out the number of clusters in noisy data set and to place prototypes correctly. Using our new definition of density, our proposed EI-Kmeans algorithm senses the noise in the data set and filters the given data set. Figure 7(b) verifies that the EI-Kmeans algorithm removes the noise and outliers from the data set. It automatically obtains the number of clusters which in this case is four and sniffs the locations of the four clusters then it initializes one prototype in the densest region of the four clusters. Figure 7(d) shows four initialized prototypes by Kmeans. The clustering result by applying Kmeans is shown in Figure 7(e) the sensitivity of Kmeans to noise and outliers collects a portion of noise as a standalone cluster and it splits one cluster into two such that each part belongs to different cluster. 
The previous figures show how we can identify the locations of the initial prototypes from the first run correctly. This means that we can able to identify the clusters in the data set without using the Kmeans algorithm. Thus we develop our new clustering algorithm called EDCA. EDCA outperforms the Kmeans algorithm in which it can identify the clusters that are not linearly separable with different sizes, shapes and densities. Figure 8(a) shows an artificial data set that contains two clusters one of them has an irregular shape that varies in density and the second one is a low density cluster. We apply EDCA a lgorithm on this data set. EDCA identifies the two clusters correctly as shown in Figure 8(b). In which we use our new formula of data point density as in (3) to specify the densest data point entire the data set. Then we start to expand the cluster from this data point which considers as a first cluster identification until we discover that there are other data points that can be specified as cluster identifications. Thus the obtained clusters are expanded at the same time until all data points in the given data set are labeled. Figures $9-11$ show the clustering results of our EDCA algorithm on different data sets. The clusters with different complex shapes, sizes and densities are identified successfully and noise and outliers are eliminated.

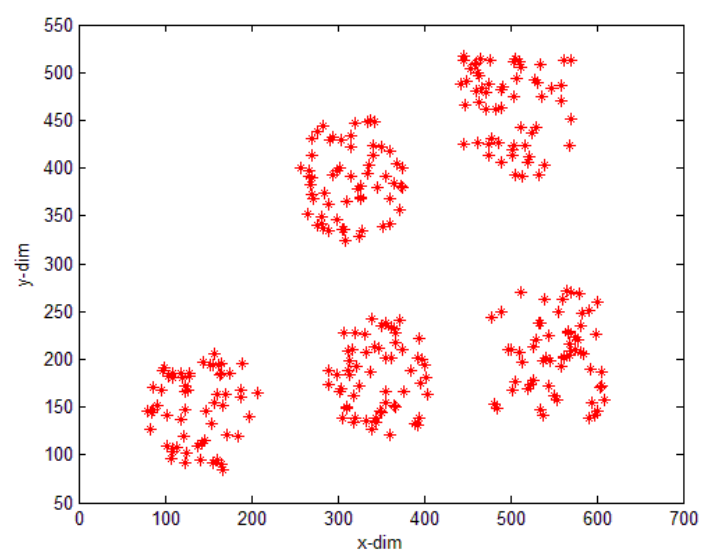

Fig. 5: (a)

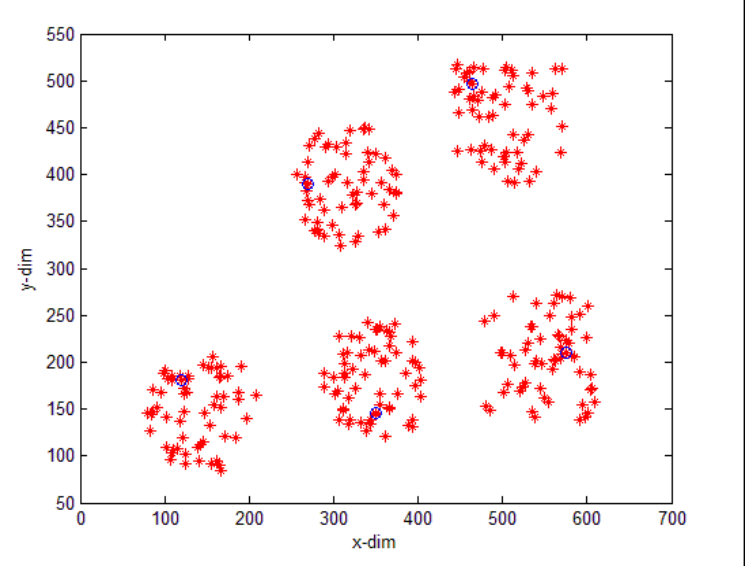

Fig. 5: (b)

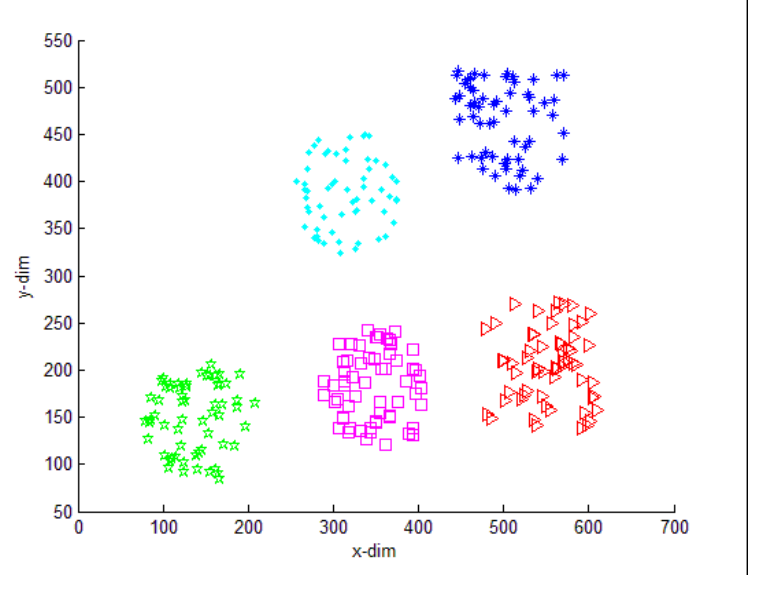

Fig. 5: (c)

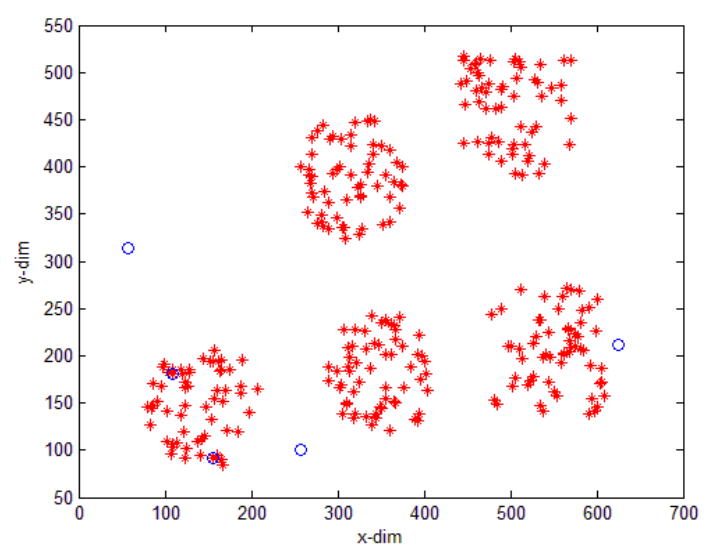

Fig. 5: (d)

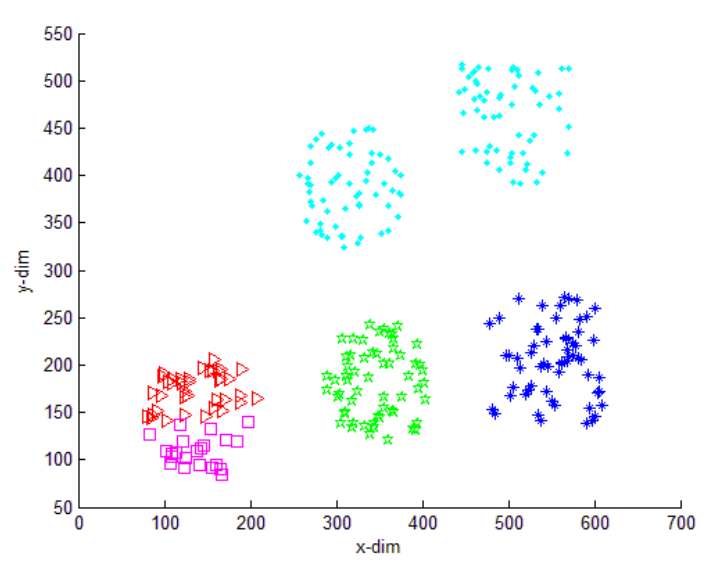

Fig. 5: (e)

Fig. 5:

(a) 300 data points with five clusters.

(b) Blue 'o's are the initialized prototypes by EI-Kmeans.

(c) Clustering result depends on the initialized prototypes in (b).

(d) Blue 'o's are the best initialized prototypes by Kmeans after 5 times running of Kmeans.

(e) Best clustering result depends on the initialized prototypes in (d) 


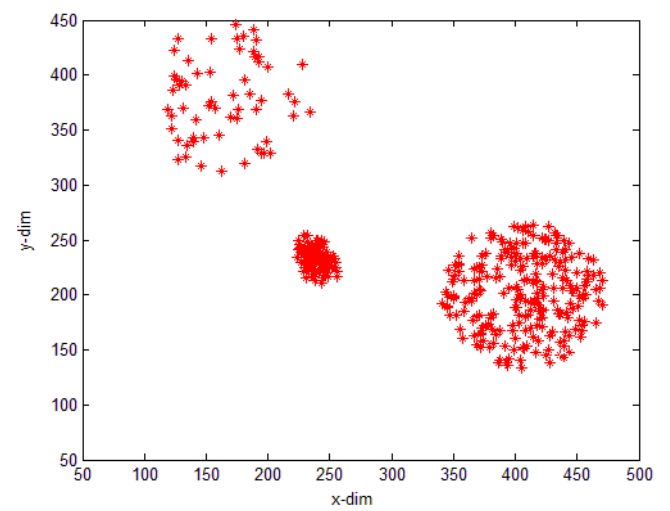

Fig. 6: (a)

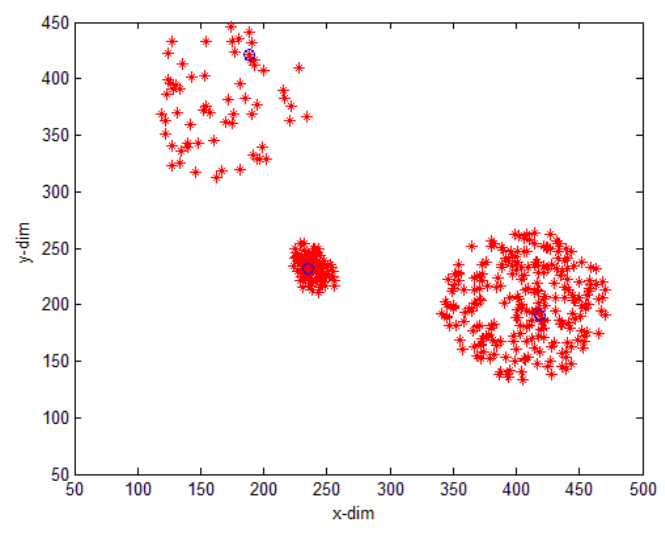

Fig. 6: (b)

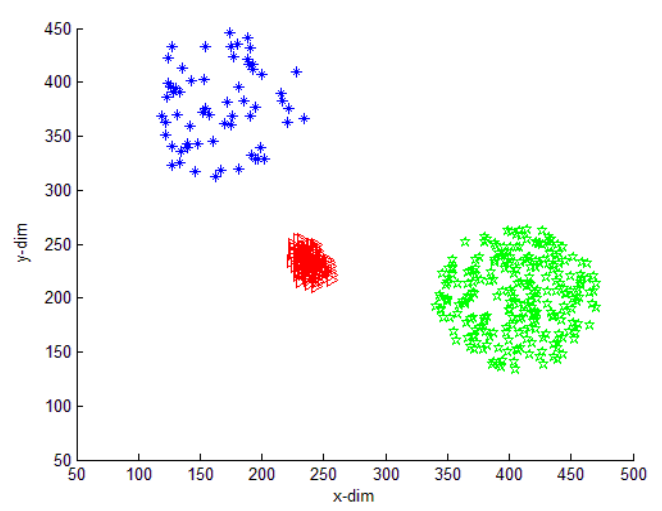

Fig. 6: (c)

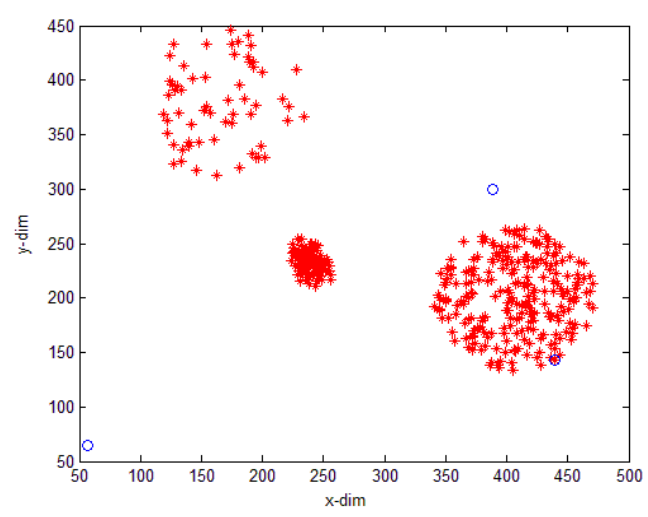

Fig. 6: (d)

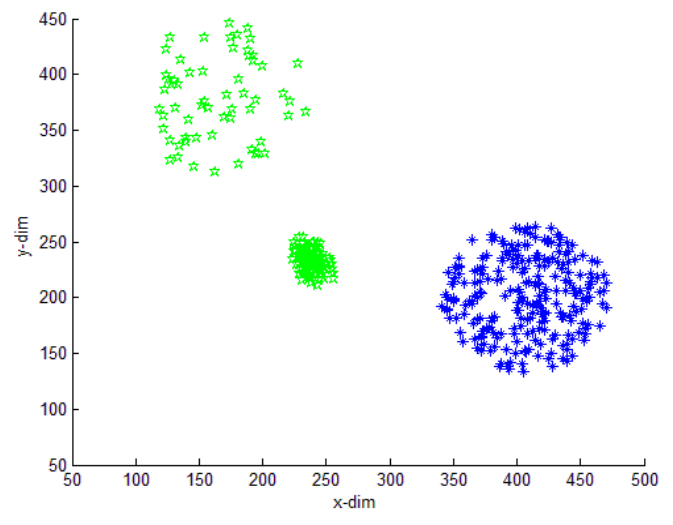

Fig. 6: (e)

Fig. 6:

(a) 400 dat a points with three clusters having different densities and sizes.

(b) Blue 'o's are the initialized prototypes by EI-Kmeans.

(c) Clustering result depends on the initialized prototypes in (b).

(d) Blue 'o's are the best initialized prototypes by Kmeans after 5 times running of Kmeans.

(e) Best clustering result depends on the initialized prototypes in (d)

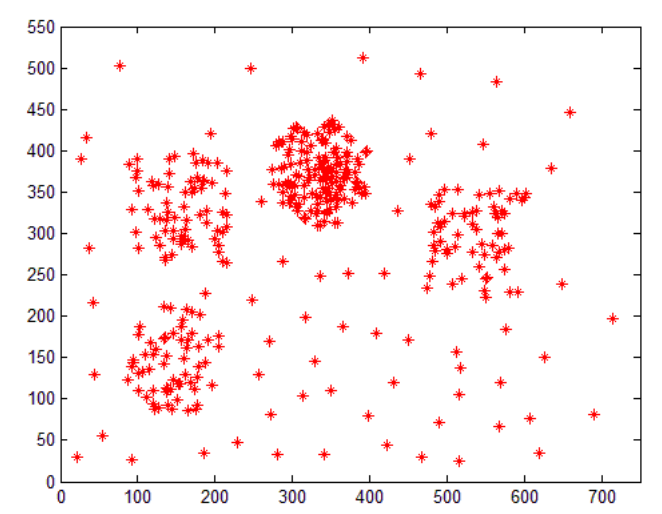

Fig. 7: (a)

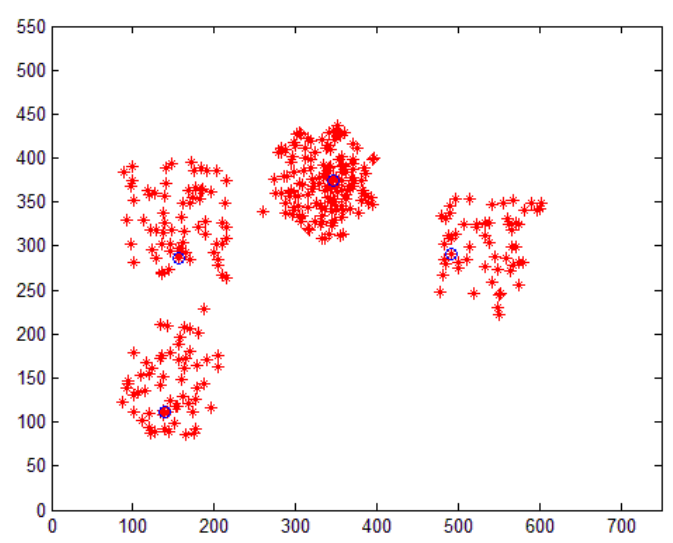

Fig. 7: (b) 


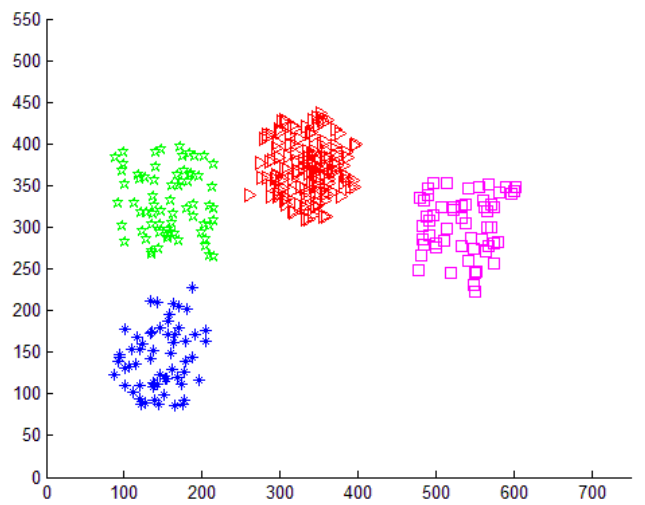

Fig. 7: (c)

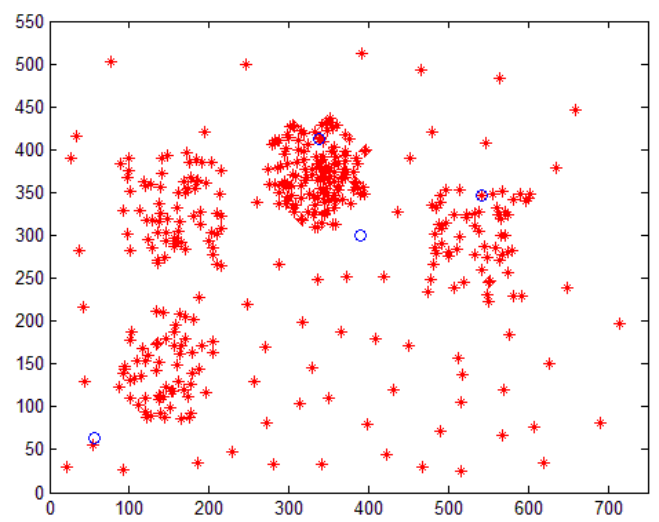

Fig. 7: (d)

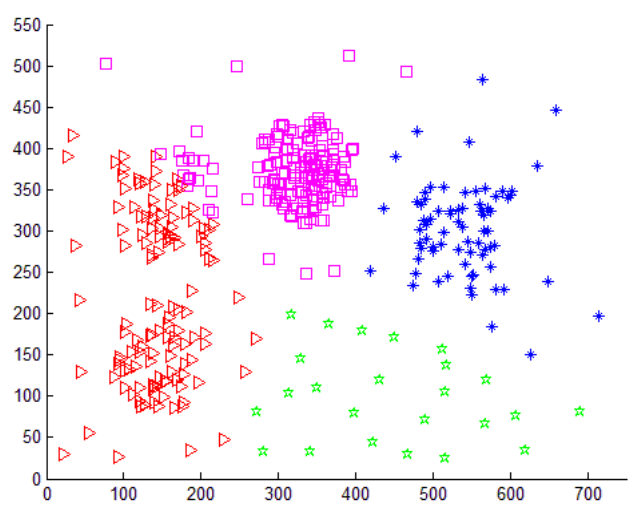

Fig. 7: (e)

Fig. 7: A Noisy data set with four clusters.

(a) Original dat a set with noise and outliers.

(b) Blue 'o's are the initialized prototypes by EI-kmeans after detecting noise and number of clusters.

(c) Clustering result depends on (b).

(d) Blue 'o's are the best initialized prototypes by k-means after 5 times running of Kmeans.

(e) Clust ering result depends on the initialized prototypes in (d)

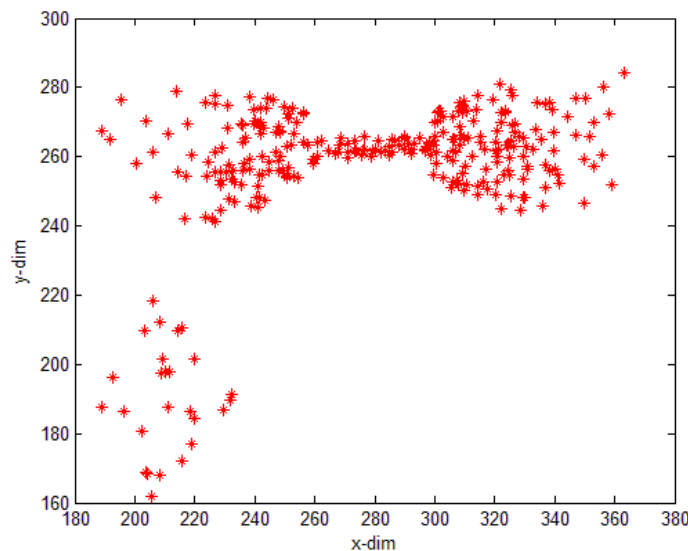

Fig. 8: (a)

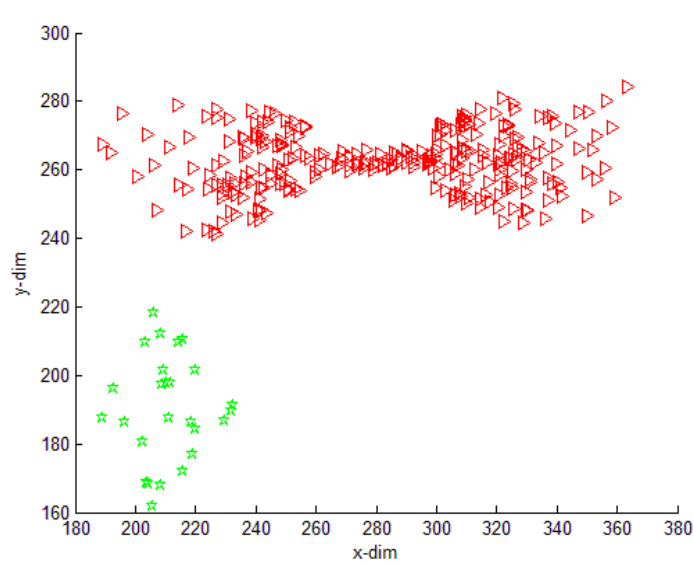

Fig. 8: (b)

Fig. 8:

(a) Original dat a set with two clusters.

(b) Clustering result of (a) using EDCA algorithm.

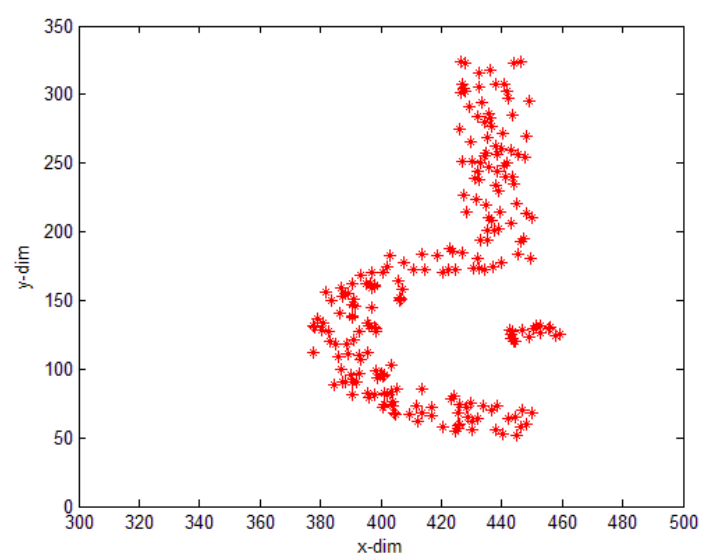

Fig. 9: (a) 


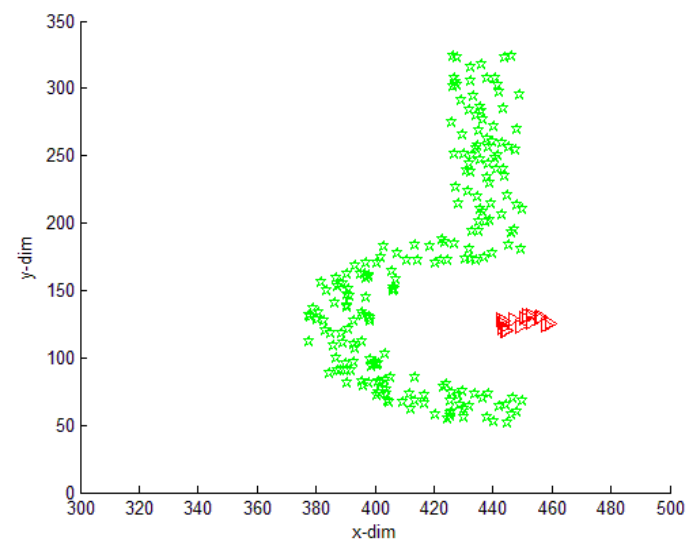

Fig. 9: (b)

Fig. 9:

(a) Original data set with two clusters.

(b) Clustering result of (a) using EDCA algorithm

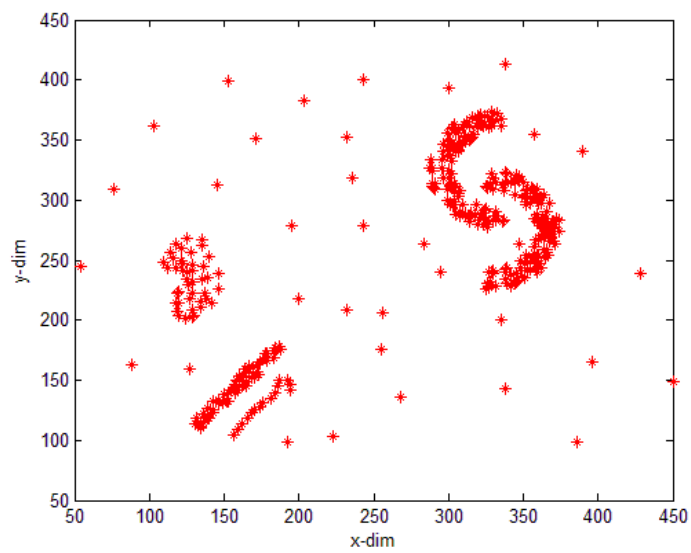

Fig. 10: (a)

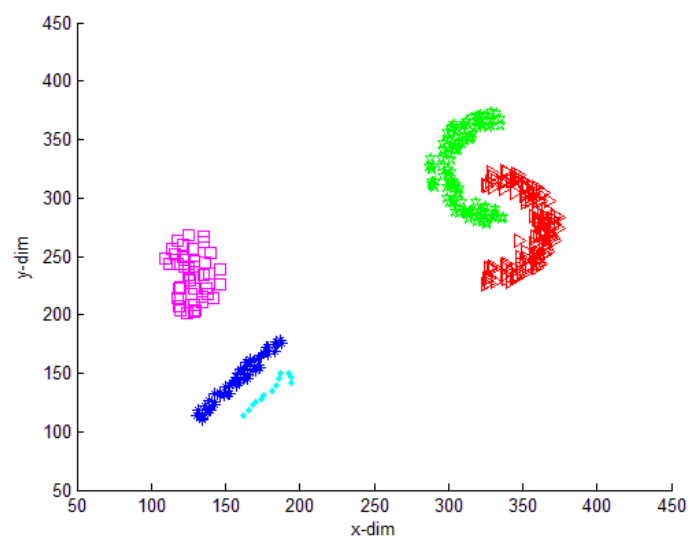

Fig. 10: (b)

Fig. 10:

(a) Original data set with five clusters including noise and outliers. (b) Clustering result of (a) using EDCA algorithm

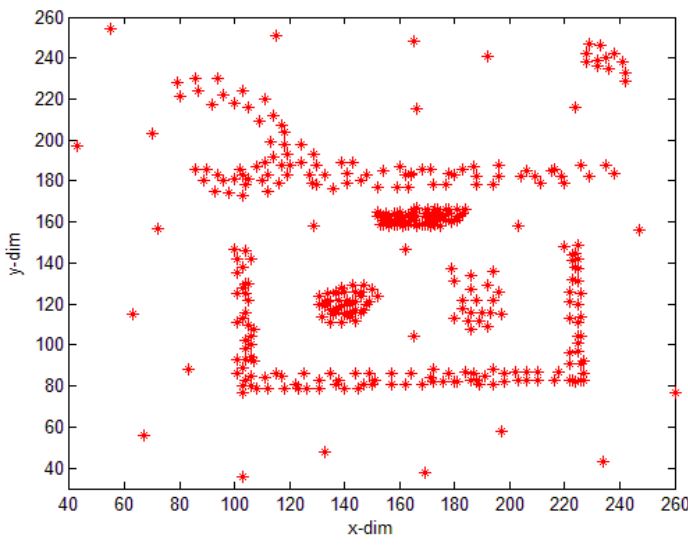

Fig. 11: (a)

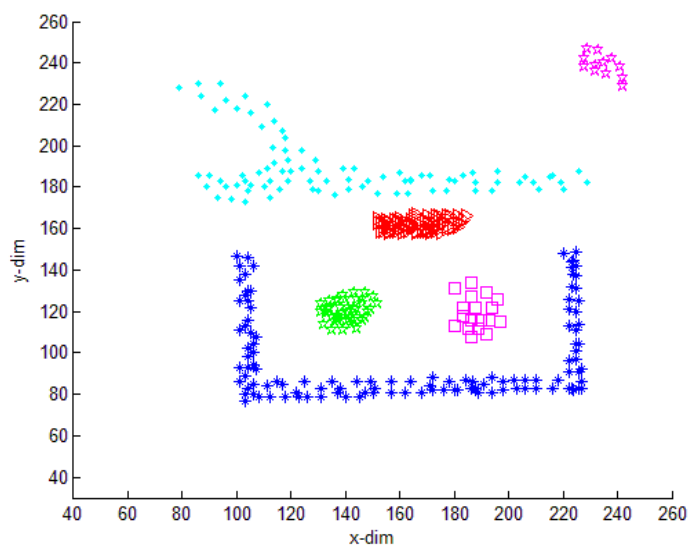

Fig. 11: (b)

Fig. 11:

(a) Original data set with six clusters including noise and outliers.

(b) Clust ering result of (a) using EDCA algorithm

Real data set: We use the iris data set from the UCI (http://archive.ics.uci.edu/ml/datasets/Iris) which contains three clusters, 150 data points with 4 dimensions. For measuring the accuracy of our proposed algorithms, we use an average error index in which we count the misclassified samples and divide it by the total number of samples. We apply the EIKmeans algorithm and obtain an error rate of $6.7 \%$. While when applying the Kmeans algorithm 7 times running we have an average error index of $38.4 \%$. The details information is shown in Table 1 . We can see that the error index of EI-Kmeans is the same in all 7 runs. This is due to the fact that the prototypes are initially calculated based on our definition of density and connectivity. Thus we obtain a competent result from the first run. On the other hand, the results for Kmeans vary from $12 \%$ to $65.3 \%$. At each run the prototypes are thrown randomly. Thus we can have some results to be close to our algorithm. So, the EI-Kmeans outperforms the Kmeans as an average. We also apply our EDCA algorith $m$ on IRIS data set and we obtain an error index of $29.3 \%$. This is due to the fact that the Iris data set has one fully separated cluster and the other two clusters 
contain some of overlapping data points. Thus the use of path connectivity should be improved to include the similarity data points' distributions in which the overlapping clusters may be distinguishable from each other according to their distributions. We apply another data set which is Glass data set form UCI (http://archive.ics.uci.edu/m1/datasets/Glass +Identificati on) to show the efficiency of our proposed algorithms.

The Glass data set contains six clusters, 214 data points with 10 dimensions. The obtained results are shown in Table 2. We get an average error index of $56.8 \%$ when we apply Kmeans 7 times. While the results for the proposed algorithms are the same in all 7 runs. The results show that the proposed EDCA algorithm has an error index of $27.6 \%$ which outperforms both Kmeans and the proposed EI-Kmeans. The Kmeans algorithm has varying results form $43.9 \%$ to $73.4 \%$. Our proposed EI-Kmeans algorithm has an improved result from the first run due to the best locations for the initialized prototypes.

Table 1: Comparison between the Results of Kmeans, our proposed EI-Kmeans and our proposed EDCA on IRIS data set

\begin{tabular}{|c|c|c|c|c|c|c|c|c|c|}
\hline \multirow{2}{*}{$\begin{array}{l}\text { \# of } \\
\text { Runs }\end{array}$} & \multicolumn{3}{|c|}{ Kmeans } & \multicolumn{3}{|c|}{ EI-Kmeans } & \multicolumn{3}{|c|}{ EDCA } \\
\hline & $\begin{array}{l}\text { \# of true } \\
\text { classified }\end{array}$ & $\begin{array}{c}\text { \# of } \\
\text { misclassified }\end{array}$ & $\begin{array}{c}\text { Error Index } \\
\%\end{array}$ & $\begin{array}{l}\text { \# of true } \\
\text { classified }\end{array}$ & $\begin{array}{c}\text { \# of } \\
\text { misclassified }\end{array}$ & $\begin{array}{c}\text { Error Index } \\
\%\end{array}$ & $\begin{array}{l}\text { \# of true } \\
\text { classified }\end{array}$ & $\begin{array}{c}\text { \# of } \\
\text { misclassified }\end{array}$ & $\begin{array}{l}\text { Error } \\
\text { Index \% }\end{array}$ \\
\hline 1 & 68 & 82 & 54.7 & 140 & 10 & 6.7 & 106 & 44 & 29.3 \\
\hline 2 & 121 & 29 & 19.3 & 140 & 10 & 6.7 & 106 & 44 & 29.3 \\
\hline 3 & 113 & 37 & 24.7 & 140 & 10 & 6.7 & 106 & 44 & 29.3 \\
\hline 4 & 64 & 86 & 57.3 & 140 & 10 & 6.7 & 106 & 44 & 29.3 \\
\hline 5 & 132 & 18 & 12 & 140 & 10 & 6.7 & 106 & 44 & 29.3 \\
\hline 6 & 97 & 53 & 35.3 & 140 & 10 & 6.7 & 106 & 44 & 29.3 \\
\hline 7 & 52 & 98 & 65.3 & 140 & 10 & 6.7 & 106 & 44 & 29.3 \\
\hline
\end{tabular}

Table 2: Comparison between the Results of Kmeans, our proposed EI-Kmeans and our proposed EDCA on GLASS data set

\begin{tabular}{|c|c|c|c|c|c|c|c|c|c|}
\hline \multirow{2}{*}{$\begin{array}{l}\text { \# of } \\
\text { Runs }\end{array}$} & \multicolumn{3}{|c|}{ Kmeans } & \multicolumn{3}{|c|}{ EI-Kmeans } & \multicolumn{3}{|c|}{ EDCA } \\
\hline & $\begin{array}{l}\text { \# of true } \\
\text { classified }\end{array}$ & $\begin{array}{c}\text { \# of } \\
\text { misclassified }\end{array}$ & $\begin{array}{c}\text { Error Index } \\
\%\end{array}$ & $\begin{array}{l}\text { \# of true } \\
\text { classified }\end{array}$ & $\begin{array}{c}\text { \# of } \\
\text { misclassified }\end{array}$ & $\begin{array}{c}\text { Error Index } \\
\%\end{array}$ & $\begin{array}{l}\text { \# of true } \\
\text { classified }\end{array}$ & $\begin{array}{c}\# \text { of } \\
\text { misclassified }\end{array}$ & $\begin{array}{l}\text { Error } \\
\text { Index \% }\end{array}$ \\
\hline 1 & 95 & 119 & 55.6 & 136 & 78 & 36.4 & 155 & 59 & 27.6 \\
\hline 2 & 85 & 129 & 60.3 & 136 & 78 & 36.4 & 155 & 59 & 27.6 \\
\hline 3 & 120 & 94 & 43.9 & 136 & 78 & 36.4 & 155 & 59 & 27.6 \\
\hline 4 & 118 & 96 & 44.8 & 136 & 78 & 36.4 & 155 & 59 & 27.6 \\
\hline 5 & 96 & 118 & 55.1 & 136 & 78 & 36.4 & 155 & 59 & 27.6 \\
\hline 6 & 76 & 138 & 64.5 & 136 & 78 & 36.4 & 155 & 59 & 27.6 \\
\hline 7 & 57 & 157 & 73.4 & 136 & 78 & 36.4 & 155 & 59 & 27.6 \\
\hline
\end{tabular}

\section{Conclusion}

We have proposed new algorithms to cope the problems of one of the most used clustering algorith $\mathrm{m}$. These problems include the bad initialized prototypes and predefined number of clusters in the standard Kmeans algorith $\mathrm{m}$. The determination of the number of clusters in the data set is considered one of the most difficult problems in data clustering. We show how the bad initialized prototypes for Kmeans can affect clustering results poorly by obtaining empty clusters, merging or splitting the clusters. Thus we define a novel density computation method of data points. So, we can efficiently define the dense region in the given data set. Which we use it to locate the prototypes in precise positions from the first run of our EI-Kmeans algorith m. Our proposed EI-Kmeans algorithm is robust to noise and outliers where it can detect noise in the data set and eliminate it from the clustering results. We define two versions of our algorith $m$ the first version takes the $k_{n}$ nearest neighbors and the number of clusters as input parameters and the second version takes only one parameter which is the k-nearest neighbors and automatically detect number of clusters in the data set. The third version of our proposed algorithm considers as a new clustering algorithm called EDCA. EDCA is based on our definition of density. Thus the proposed EDCA focuses on the limitation of the Kmeans clustering itself. In which it outperforms the Kmeans in finding clusters of non-globular shapes, different sizes and densities. Our simulation results show the efficiency of the proposed algorithms.

\section{References}

[1] M. Eirinaki and M. Vazirgiannis, 2003. Web Mining for Web Personalization, in ACM Transactions on Internet Technology (TOIT), vol. 3, no. 1 pp: 1-27. DOI: 10.1145/643477.643478

[2] B.Bahmani Firouzi, T. Niknam, and M. Nayeripour, Dec 2008. A New Evolutionary Algorithm for Cluster Analysis, Proc. of world 
Academy of Science, Engineering and Technology, vol. 36. http://www.waset.org/journals/waset/v46/v46100.pdf

[3] Gersho and R. Gray, 1992. Vector Quantization and Signal Compression, Kulwer Acadimec, Boston.http://www-ee.stanford.edu/ gray/

[4] M. A1- Zoubi, A. Hudaib, A. Huneiti and B. Hammo, 2008. New Efficient Strategy to Accelerate k-Means Clustering Algorithm, American Journal of Applied Science, vol. 5, no. 9 pp: 1247-1250. DOI: 10.3844/ajassp.2008.1247. 1250

[5] M. Celebi, 2009. Effective Initialization of Kmeans for Color Quantization, Proc. of the IEEE International Conference on Image Processing, pp: 1649-1652. DOI: 10.1.1.151.5281

[6] M. Borodovsky and J. McIninch, 1993. Recognition of genes in DNA sequence with ambiguities, Biosystems, vol. 30, issues 1-3, pp: 161-171. DOI:10.1016/0303-2647(93)90068-N

[7] J. Bezdek and N. Pal, 1992. Fuzzy Models for Pattern Recognition, IEEE press (New York, NY, USA). http://www.amazon.co m/Fu zzy -Models Pattern-Recognition-Structures/dp/0780304225

[8] J. Bezdek, 1981. Pattern Recognition with Fuzzy Objective Function Algorithms, Plenum Press (New York, NY, USA). http://www.a mazon.co m/Recognition-ObjectiveFunction-Algorithms-Applications/dp/0306406713

[9] G. Gan, Ch. Ma, and J. Wu, 2007. Data Clustering: Theory, Algorith ms, and Applications, ASA-SIAM series on Statistics and Applied Probability, SIAM. DOI: $10.1111 /$ j.1751-5823.2007.00039_2.x

[10] D. Defays, 1977. An Efficient Algorithm for A Complete Link Method, The Computer Journal, vol. 20, pp: 364-366. DOI: 10.1093/comjn1/20.4.364

[11] R. Sibson, 1973. SLINK: an Optimally Efficient Algorith m for the Single Link Cluster Method, The Computer Journal, vol. 16, No. 1, pp: 30-34. DOI: 10.1093/comjn1/16.1.30

[12] L. Kaufman, and P. Rousseeuw, 1990. Finding Groups in Data: An Introduction to Cluster Analysis, (John Wiley \& Sons). DOI: 10.1002/9780470316801

[13] J. MacQueen, 1967. Some Methods for Classification and Analysis of Multivariate Observations, 5th Berkeley Symp. Math. Statist. Prob., vol. 1, pp: 281-297. http://digitalassets.lib.berkeley.edu/math/ucb/text/ math_s5_v1_article-17.pdf

[14] U. Fayyad, G. Piatetsky-Shapiro, P. Smyth, R. Uthurusamy, 1996. From Data Mining to
Knowledge Discovery in Databases, Advances in Knowledge Discovery and Data Mining, AAAI/MIT press. DOI: 10.1.1.42.1071

[15] XindongWu and et. Al., 2008. Top 10 Algorithms in Data Mining, Journal of Knowledge and Information Systems, vol. 14. Issues 1-37. DOI: $10.1007 / \mathrm{s} 10115-007-0114-2$

[16] I.S. Dhillon, Y. Guan, and B. Kulis, 2004. Kernel Kmeans, Spectral Clustering and Normalized Cuts. ACM SIGKDD international conference Knowledge Discovery and Data Mining. (Seattle, WA). DOI: 10.1.1.79.2967

[17] M. Girolami, 2002. Mercer Kernel Based Clustering in Feature Space. IEEE Transactions on Neural Networks, 13(3), pp: 780-784. DOI:10.1.1.135.4576

[18] Scholkopf, A. Smola, and K.R. Muller, 1998. Nonlinear Component Analysis As a Kernel Eigenvalue Problem. Neural Computation (10), pp: 1299-1319. DOI: 10.1.1.100.3636

[19] J. Suykens, and J. Vandewalle, 1999. Least Squares Support Vector Machine Classifiers. Neural Processing Letters, 9(3), pp: 293-300. DOI: 10.1.1.7.2877

[20] W. Barbakh, and C. Fyfe, 2008. Clustering and Visualization with Alternative Similarity Functions The 7th WSEAS international conference on artificial intelligence, knowledge, engineering and data bases, AIKED'08 (University of Cambridge, UK) pp: 238-244. http://www.wseas.us/elibrary/conferences/2008/uk/AIKED/AIKED34.pdf

[21] W. Barbakh, and C. Fyfe, 2008. Online Clustering Algorithms. International Journal of Neural Systems (IJNS), 18(3), pp: 185-194. http://www.uws.ac.uk/schoolsdepts/computing/doc uments/fyfe-online-clustering-algorithms.pdf

[22] R. Duda, P. Hart, and D. Stork, 2001. Pattern Classification, (John Wiley \& Sons, second edition). DOI: 10.1007/s00357-007-0015-9

[23] N. Asgharbeygi, and A. Maleki, 2008. Geodesic Kmeans Clustering, 19th International Conference on Pattern Recognition. http://www.stanford.edu/ arianm/geo_kmeans_icp r08.pdf

[24] M. Ester, H. P. Kriegel, J. Sander, and X. Xu, 1996. A Density-based Algorithm for Discovering Clusters in Large Spatial Data sets with Noise, 2nd International Conference on Knowledge Discovery and Data Mining, pp: 226-231. DOI: 10.1.1.121.9220

[25] E. Forgy, 1965. Cluster Analysis of Multivariate Data: Efficiency vs. Interpretability of Classification, Biometrics, vol. 21. 
http://www.citeulike.org/user/dgrianna/article/3382 461

[26] D. Hochbaum, and D. Shmoys, 1985. A Best Possible Heu ristic for the K-Center Problem, Math. Oper. Res., vol. 10, no. 2, pp: 180-184. DOI: 10.1287/moor.10.2.180

[27] D. Arthur, and S. Vassilvitskii, 2007. Kmeans++: The Advantages of Care ful Seeding, Proceeding of SODA'07, pp: 1027-1035. DOI: $10.1145 / 1283383.1283494$

[28] M. Al-Daoud, 2005. A New Algorithm for Clustering Initialization, Proceeding World Academy of Science, Engineering, and Technology, vol. 4. http://www.waset.org/journals/waset/v4/v4-20.pdf

[29] W. Barbakh, and C. Fyfe, 2008. Local vs. Global Interactions in Clustering Algorithms: Advances over Kmeans, International Journal of knowledgebased and Intelligent Engineering Systems, vol. 12. http://ios press.metapres s.com/content/6443723640 674366/

[30] W. Barbakh and C. Fyfe, 2007. Inverse Weighted Clustering Algorithm, Computing and Information Systems, 11(2), pp: 10-18, ISSN 1352-9404. http://cis.uws.ac.uk/research/journal/V11n2/IW C.p df

[31] W. Barbakh, 2007. The Family of Inverse Exponential Kmeans Algorithms, Computing and Information Systems, 11(1), pp: 1-10. ISSN 13529404.

http://cis.uws .ac.uk/research/journal/vol11.htm

[32] Jim Z.C. Lai, and T. J. Huang, 2010. Fast Global Kmeans Clustering Using Cluster Membership and Inequality, Pattern Recognition, vol. 43, pp: 19541963. DOI:10.1016/j.patcog.2009.11.021

[33] G. Frahling, and Ch. Sohler, 2008. A Fast Kmeans Implementation Using Coresets, International Journal of Computational Geometry and Applications, vol. 18, Issue 6, pp: 605-625. DOI: 10.1145/1137856.1137879

[34] L. Taoying, and Y. Chen, 2008. An Improved Kmeans for Clustering Using Entropy Weighting measures, 7th World Congress on Intelligent Control and Automation. DOI: 10.1109/WCICA.2008.4592915

[35] S. Gupara, K. Rao, and V. Bhatnagar, 1999. Kmeans Clustering Algorithm for Categorical Attributes, Proceeding 1st International Conf. on Data Werehousing and Knowledge Discovery, pp: 203-208. DOI: 10.1.1.24.9576

[36] Z. Huang, 1998. Extensions to The Kmeans Algorithms for Clustering Large Data Sets with Categorical Values, Data Mining Knowledge Discovery, vol. 2, pp: 283-304. DOI: 10.1.1.15.4028

\section{Authors' Profiles}

Mohamed Abubaker has received his B.Sc. degree in Computer Engineering in 2005 from Yarmouk University (YU) in Jordan. In 2011, he has completed his M.Sc. in Computer engineering from the Islamic University of Gaza in Palestine. In 2004 he worked as a researcher and developer at ENIC telecom Lille 1 in France for implementing and verifying the Packet-EModel. His research interests include multi-agent systems where he developed the YU soccer game simulator which simulates an intelligent collaborative robot in soccer game. He focuses on artificial intelligence, computer vision and mobile computing.

Wesam Ashour is an assistant professor at the Islamic University of Gaza. He is an active researcher at the Applied Computational Intelligence Research Unit in the University of the West of Scotland. He got his Master and Doctorate degrees from UK. His research interests include data mining, artificial intelligence, reinforcement learning and neural networks.

How to cite this paper: Mohamed Abubaker, Wesam Ashour,"Efficient Data Clustering Algorithms: Improvements over Kmeans", International Journal of Intelligent Systems and Applications (IJISA), vol.5, no.3, pp.37-49, 2013.DOI: 10.5815/ijisa.2013.03.04 